\title{
CAN MEAN HELICITY FLUCTUATIONS EXPLAIN THE VARIABILITY OF THE SOLAR CYCLE?
}

\author{
P. HOYNG \\ Laboratory for Space Research \\ Sorbonnelaan 2, 3584 AW Utrecht \\ The Netherlands \\ E-mail: phoyng@sron.ruu.nl
}

December 3, 1992

\begin{abstract}
I consider the effect of rapid fluctuations in the mean helicity on a plane dynamo wave in the $\alpha \omega$-approximation and in the weak forcing limit. The phase shift and the logarithmic amplitude of the wave exhibit a correlated random walk, so that weaker (stronger) cycles last longer (shorter). The solar cycle data follow this prediction rather well. Mean helicity fluctuations are concluded to be an important source of solar cycle variability.
\end{abstract}

Key words: mean field theory - helicity fluctuations - solar cycle - random forcing

\section{Introduction}

Dynamos modeled by linear mean field theory are strictly periodic under conditions of marginal stability. This strict periodicity ceases to exist when nonlinear effects are taken into account. Even if this is not done, there is a source of period and amplitude variability which has not been considered until recently. I employ the usual dynamo equation

$$
\frac{\partial<\mathbf{B}>}{\partial t}=\nabla \times(\mathbf{v} \times+\alpha-\beta \nabla \times)<\mathbf{B}>.
$$

Here, $\mathbf{v}$ is the mean flow and $\alpha$ and $\beta$ are the usual dynamo coefficients (see e.g. Moffatt 1978). I interpret $<>$ as a longitudinal average (Braginskii 1965). In that case, $\alpha$ and $\beta$ are also defined as longitudinal averages, and hence they must exhibit temporal fluctuations since there is only a finite number of eddies along a circle of constant radius $r$ and latitude $\theta$. Here I consider only fluctuations in $\alpha$ :

$$
\alpha=\alpha_{0}+\delta \alpha(t) ; \quad \beta=\beta_{0},
$$

and $\beta_{0}$ is regarded as a constant, while the fluctuations $\delta \alpha(t)$ are taken to be independent of position.

In this paper I study period and amplitude variations in the mean field caused by $\delta \alpha(t)$ in a simple dynamu model. An important motivation is that nonlinear mean field models suggest that there are only attractors of very high dimension. The behaviour of such a system is stochastic and may be studied by stochastic random forcing. Another source of inspiration is the work of Barnes et al. (1980) who simulated remarkably 'real' sunspot cycles from narrow band Gaussian noise. The present work is an extension of their model in the sense that a dynamo wave is used as the oscillator. For a completely different approach to the effect of fluctuations I refer to Van Geffen (1993). 


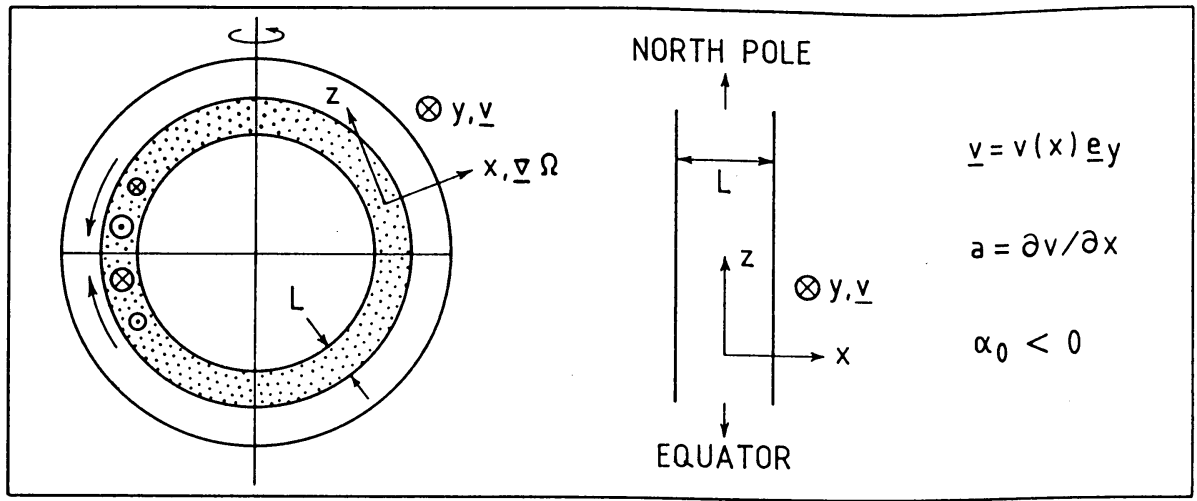

Fig. 1. Traveling dynamo waves in a boundary layer between the convection zone and the radiative interior of the Sun. For simplicity, we consider an infinite homogeneous plane layer of thickness $L$. The mean flow is along the $y$-direction and has a constant shear $a$

\section{Model}

I consider a single plane dynamo wave in a plane layer of thickness $L$, see Fig. 1 . The wave propagates in the $-z$ direction, and is represented by

$$
<\mathbf{B}>=\nabla \times A \mathbf{e}_{y}+B \mathbf{e}_{y},
$$

with

$$
\begin{aligned}
A & =P \cos \left(k_{z} z-\omega t+\varphi_{0}+\delta \chi\right) \sin \pi x / L, \\
B & =k T \cos \left(k_{z} z-\omega t+\delta \psi\right) \sin \pi x / L, \\
k & =\sqrt{k_{z}^{2}+(\pi / L)^{2}} .
\end{aligned}
$$

Here, $k_{z} z-\omega t$ is the phase, $k$ the wave number and $\varphi_{0}$ the phase difference between the poloidal and toroidal field of the unperturbed wave. The amplitudes of poloidal and toroidal field are $P$ and $T$, respectively. The fluctuations $\delta \alpha(t)$ cause the amplitudes $P$ and $T$ and the phase differences $\delta \chi$ and $\delta \psi$ to become random functions of time. There are no boundary conditions, and I really consider a plane wave in infinite space, with wave numbers $k_{z}$ and $\pi / L$ in the $z$-and $x$-direction, respectively. This simplifies the problem considerably.

Choudhuri (1992) has studied the effect of fluctuations in this model numerically. However, the model is sufficiently simple to allow a complete theoretical analysis by stochastic methods (Hoyng 1993). Random forcing by helicity fluctuations has also been studied in a simple spherical geometry (Hoyng and Van Geffen 1993).

\section{Method}

The first step in the analysis is to find the properties of the unperturbed, marginally stable wave, when $\delta \alpha(t)=0$. In that case, $P$ and $T$ are constant and $\delta \chi=\delta \psi=0$. I restrict myself to the $\alpha \omega$-limit, which means that the two dynamo numbers 


$$
d_{\alpha} \equiv \alpha_{0} / \beta_{0} k \quad \text { and } \quad d_{\omega} \equiv a k_{z} / \beta_{0} k^{3}
$$

satisfy $\left|d_{\alpha}\right| \ll 1$ and $\left|d_{\omega}\right| \gg 1$. Inserting (3), (4) and (5) in (1) leads to

$$
P_{0} / T_{0}=d_{\alpha} ; \quad d_{\alpha} d_{\omega}=2 ; \quad \varphi_{0}=\pi / 4 ; \quad \omega=\beta_{0} k^{2} .
$$

Here, $P_{0}$ and $T_{0}$ are the unperturbed wave amplitudes. For details on the derivation of (8) I refer to Hoyng (1993). The next step is to write $\alpha$ from (2) as

$$
\alpha=\alpha_{0}(1+\tilde{\alpha}), \quad \text { i.e. } \quad \tilde{\alpha}(t)=\delta \alpha(t) / \alpha_{0},
$$

and to define the random phase difference $\delta$ and the relative amplitude variation $\epsilon$ :

$$
\delta \equiv \delta \chi-\delta \psi ; \quad \epsilon \equiv \frac{P / P_{0}}{T / T_{0}}-1
$$

From now on, $\epsilon, \delta, T$ and $\delta \psi$ are treated as the independent variables. They turn out to obey the following simple equations (Hoyng 1993):

$$
\begin{array}{ll}
\dot{\epsilon}+2 \epsilon=-2 \delta+\tilde{\alpha} & \dot{T} / T=\delta+\epsilon \\
\dot{\delta}+2 \delta=2 \epsilon-\tilde{\alpha} & (\delta \psi)^{\circ}=\delta-\epsilon
\end{array}
$$

Time is now measured in units of the diffusion time $\left(\beta_{0} k^{2}\right)^{-1}$, and represents differentiation with respect to the dimensionless time $t \beta_{0} k^{2}$. Eqs. (11) are valid provided ${ }^{1}$ $\tilde{\alpha}_{\text {r.m.s. }} \sqrt{\tau_{\mathrm{c}}} \ll 1$, where $\tau_{\mathrm{c}}$ is the correlation time of $\tilde{\alpha}(t)$ in units of $\left(\beta_{0} k^{2}\right)^{-1}$. This is called the weak forcing limit. It is assumed that $\tau_{\mathrm{c}} \ll 1$. The equations for $\epsilon$ and $\delta$ form a closed set with an additive noise term. When the initial condition $c(0)$, $\delta(0)$ has died out, i.e. for $t>1$, the solution may be written as

$$
z(t)=\int_{0}^{\infty} \exp (-\lambda s) f(t-s) \mathrm{d} s,
$$

with

$$
z \equiv \epsilon+\mathrm{i} \delta ; \quad \lambda=2-2 \mathrm{i} ; \quad f(t)=(1-\mathrm{i}) \tilde{\alpha}(t) .
$$

It is straightforward to generate a numerical solution of Eqs. (11a,b), see Fig. 2. Eq. (12) is a good starting point to determine the statistical properties of $\epsilon$ and $\delta$, and, via $(11 \mathrm{~b})$, those of the phase shift $\delta \psi$ and the $\log$ arithmic amplitude $\log T$ of the toroidal field. Note that according to Eq. (11b) $T$ cannot change sign.

\section{Results}

The properties of the solutions such as those in Fig. 2 can be summarised as follows (Hoyng 1993):

1. Both $\log T$ and the phase shift $\delta \psi$ of the toroidal field perform a random walk with the same diffusion coefficient $D$ :

$$
\begin{aligned}
& (\log T)_{\text {r.m.s. }}=\delta \psi_{\text {r.m.s. }} \simeq \sqrt{2 D t} ; \quad D=\frac{1}{4} \tilde{\alpha}_{\text {r.m.s. }}^{2} \tau_{\mathrm{c}}, \\
& { }^{1} x_{\text {r.m.s. }} \equiv\left\langle x^{2}\right\rangle^{1 / 2} \text { for any zero-mean random variable } x .
\end{aligned}
$$






Fig. 2. Irregular cycles of an $\alpha \omega$-dynamo randomly forced by mean helicity fluctuations. Plotted is $\{T \cos (\delta \psi-\omega t)\}^{2}$ (the square of the toroidal field strength, apart from irrelevant phase factors) versus time in units of the unperturbed dynamo period $2 \pi / \omega$. Parameters: $\tilde{\alpha}_{\text {r.m.s. }} \sqrt{\tau_{\mathrm{c}}}=0.218 ; T(0)=1 ; \delta \psi(0)=\pi / 2 ; \epsilon(0)=\delta(0)=0$

and $D \ll 1$ due to the assumption of weak forcing. The implication is that the amplitude $T$ reaches any desired order of magnitude if we wait long enough.

2. The random walks of $\log T$ and $\delta \psi$ are completely anticorrelated:

$$
\log T+\delta \psi=\text { const. }+F(t) ; \quad F_{\text {r.m.s. }}=\sqrt{D} \ll 1 .
$$

$F(t)$ is a noise term of constant r.m.s. magnitude. The constant in (15) depends on the initial conditions. Eq. (15) implies that cycles of shorter/longer duration have larger/smaller amplitudes. This effect is clearly visible in Fig. 2, and is also observed in the solar cycle (Waldmeier 1935; Ioyng 1993).

3. The quality factor of the oscillator, defined as $Q=\omega / \Delta \omega$ where $\Delta \omega$ is the width of the line in the power spectrum, is given by

$$
Q=D^{-1}
$$

4. The poloidal field follows the behaviour of the toroidal field since $\epsilon_{\text {r.m.s. }}=$ $\delta_{\text {r.m.s. }} \sqrt{3}=\sqrt{3 D} \ll 1$.

\section{Application to the solar dynamo}

To verify to what extent the solar cycle follows the predicted correlation (15) I use the epochs $t_{k}$ of sunspot maxima and the corresponding sunspot numbers $R_{k}$ (Allen 1973) to define the phase shift at the $k$-th maximum and $\log T_{k}$ :

$$
\begin{aligned}
\delta \psi_{k} & =\bar{\omega}\left(t_{k}-t_{1}\right)-(k-1) \pi, \\
\log T_{k} & =\mu \log \left(R_{k} / R_{1}\right) .
\end{aligned}
$$

In this way, $\delta \psi_{1}=\log T_{1}=0$ at $t_{1}=1705.5$. Here, $\bar{\omega}$ is the unknown frequency of the solar dynamo. The sunspot numbers $R_{k}$ are assumed to be proportional to some power of $T_{k}$, and I write $T_{k} \propto R_{k}^{\mu}$. A least square fit of (17) and (18) to rela- 


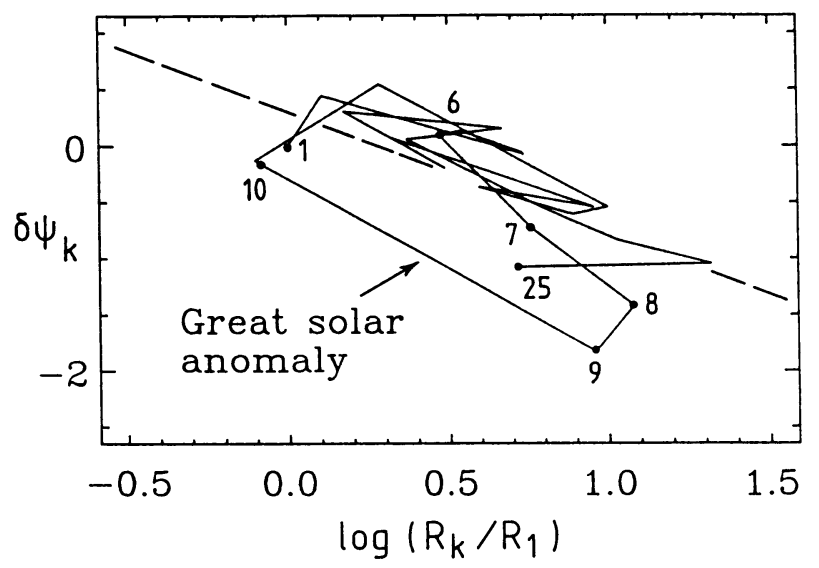

Fig. 3. Plot of $\delta \psi_{k}$ defined in (18) versus $\log \left(R_{k} / R_{1}\right)$ for the parameters of the best fit. The dashed line is the best correlation line, and is given by $\delta \psi+1.07 \log \left(R / R_{1}\right)=0.3$. The labels 1 and 25 indicate the first and last data point. The points labeled 6 through 10 comprise what Dicke (1988) refers to as the 'great solar anomaly'

tion (15) gives $2 \pi / \bar{\omega}=22.4 \mathrm{yr}$ and $\mu=1.07$ (plus the irrelevant value of the constant in (15)). Furthermore I find that $Q \approx F_{\text {r.m.s. }}^{-2} \approx 10$. The result of the fit is shown in Fig. 3. The anticorrelation between $\delta \psi$ and $\log T$ is rather convincing.

\section{Conclusions}

I conclude that fluctuations in the mean helicity are a promising mechanism to explain the observed solar cycle variability. From $Q \approx 10$ we infer that the fluctuations are large, $\delta \alpha_{\text {r.m.s. }} /\left|\alpha_{0}\right| \approx 5$ for $\tau_{\mathrm{c}}=15$ days. The fact that $\mu \simeq 1$ implies that the sunspot numbers are directly proportional to the mean field strength. Model simulations (Hoyng 1993) show many features also seen in the real cycle (quasiperiodicity, amplitude modulation, recurrent long minima, etc.). However, nonlinear effects must be included to prevent an unrestricted drift of the amplitude.

\section{References}

Allen, C.W.: 1973, Astrophysical Quantities, The Athlone Press, London

Barnes, J.A., Sargent, H.H. and Tryon, P.V.: 1980, in Pepin, R.O., Eddy, J.A. and Merrill, R.B., ed(s)., The Ancient Sun, Pergamon Press, New York, p. 159

Braginskii, S.I.: 1965, Sov. Phys. JETP 20, 726

Choudhuri, A.R.: 1992, Astron. Astrophys. 253, 277

Dicke, R.H.: 1988, Solar Phys. 115, 171

Hoyng, P.: 1993, Astron. Astrophys. , in press

Hoyng, P. and Van Geffen, J.H.G.M.: 1993, Geophys. Astrophys. Fluid Dynam. , in press

Moffatt, K.H.: 1978, Magnetic Field Generation in Electrically conducting Fluids, Cambridge University Press, Cambridge

Van Geffen, J.H.G.M.: 1993, Magnetic Energy Balance and Period Stability of the Solar Dynamo, Thesis, Univ. of Utrecht

Waldmeier, M.: 1935, Astr. Mitt. Zürich 14 No. 133, 105 